\title{
PERCEPCIÓN SUBJETIVA DEL ESPACIO EN DEPORTISTAS SELECCIONADOS DE LAS UNIVERSIDADES TRADICIONALES DE LA REGIÓN DE VALPARAISO, CHILE
}

\section{SUBJECTIVE PERCEPTION THE SPREAD IN SPORTSMEN SELECTED OF THE TRADITIONAL UNIVERSITIES OF THE REGION VALPARAISO, CHILE}

\author{
Paez Herrera, Jacqueline Del Carmen \\ Escuela de Educación Física, Pontificia Universidad Católica de Valparaíso.
}

\begin{abstract}
PAEZ H.J. Percepción subjetiva del espacio en deportistas seleccionados de las universidades tradicionales de la región de Valparaiso, Chile. Mot. Hum., 10(2): 103-110, 2010.

\section{RESUMEN}

La Percepción Subjetiva de Espacio (PSE), ha sido considerada como una de las capacidades determinantes en el rendimiento deportivo, que se ve reflejada, en un desarrollo del juego más rápido y preciso en un ambiente de grandes niveles de incertidumbre temporal y espacial. Es por esto que esta investigación tiene como fin conocer el nivel de PSE en dos situaciones diferentes, en deportistas seleccionados universitarios de la V Región. La investigación es de tipo descriptiva, con un paradigma empírico-analítico. La población fue constituida por $N=130$ deportistas $(n=48$ damas y $n=$ 82 varones). Para determinar la variable dependiente se utilizó el test PSE, con una modalidad de distancia fija y distancia a determinar. Los principales resultados una vez aplicada la prueba $t$ student indican que: a) no existen diferencias significativas en los deportistas universitarios en los desempeños del test PSE-1 y PSE-2. b) en el TPSE-2 en relación a la comparación de sexos existen diferencias significativas $(p<0.05)$
\end{abstract}

Palabras Claves: Percepción Subjetiva, Tiempo, Espacio, Deportistas, Universitarios.

\section{INTRODUCCION}

En la práctica deportiva encontramos diferentes formas en cuanto a su función y ejecución, las que pueden ser organizadas o no. En relación a esto, se identifican diferentes tipos de práctica deportiva, las cuales pueden ser muy diferentes entre si y tener objetivos radicalmente distintos, pero todas necesitan para un buen rendimiento motriz ciertas capacidades del ejecutante, además de ciertas condiciones para su ejecución. Cuando todos los elementos se ponen al servicio de los deportistas, nos encontramos con el hecho DE que cualquier deporte en cuestión gana en rendimiento. Para poder alcanzar una adecuada respuesta motriz que conlleve a un buen rendimiento, el deportista se ve enfrentado a diferentes situaciones y una de ellas es cuando su acción se debe realizar en contextos con grandes niveles de incertidumbre (presencia de balón, compañeros, oponentes), y entonces debe seleccionar la acción más adecuada en el momento más adecuado, en el que el rendimiento dependerá tanto de una correcta percepción de los estímulos que se dan durante el juego, de la correcta y rápida decisión de la respuesta a emitir, así como de la habilidad para ejecutar esa respuesta seleccionada.
El presente estudio se desarrollará en el área deportiva estructurada como son los deportes Colectivos e Individuales. Dentro de los Deportes Colectivos se estudia la práctica de deportes como el, Básquetbol y Vóleibol; y en lo que concierne a los deportes Individuales, el Tenis de Mesa y los deportes de Combate, en damas y varones.

Tiene como propósito realizar un acercamiento reflexivo a la capacidad de percepción de espacio, en la ejecución de dos test motores en deportistas seleccionados de los deportes mencionados. Estas capacidades se estudian en una situación experimental creada y validada, para tal fin. A partir de este estudio se pretende conocer más acerca de uno de los factores que interfieren en el rendimiento deportivo: la influencia de la capacidad de percepción de espacio en los deportistas en función con la ejecución motora dentro de cualquier situación deportiva competitiva.

Desde de estas intenciones, se plantean las siguientes interrogantes de estudio ¿Cuál es la capacidad perceptiva de espacio de los deportistas que practican los deportes en estudio? ¿Será que la 
PAEZ H.J. Percepción subjetiva del espacio en deportistas seleccionados de las universidades tradicionales de la región de Valparaiso, Chile. Mot. Hum., 10(2): 103-111, 2010.

PSE en el ámbito deportivo se encuentra diferencia entre los deportes colectivos e individuales en la capacidad de percepción subjetiva de espacio ?.

\section{MATERIAL Y METODOS}

Fueron medidos, registrados y analizados alumnos universitarios seleccionados de deportes colectivos e individuales (todos bajo techo), de las universidades influenciada por el factor género? ¿Existe tradicionales dependientes del Consejo de Rectores de la Región de Valparaíso, es decir, Pontificia Universidad Católica de Valparaíso (PUCV), Universidad Federico Santa María (UFSM), Universidad de Valparaíso (UV) y Universidad Playa Ancha (UPLA). Los datos de los seleccionados participantes son presentados en la TABLA I, considerando las variables universidad, deportes y género, con un $n=130$.

\begin{tabular}{|c|c|c|c|c|c|c|c|c|c|c|c|}
\hline \multicolumn{12}{|c|}{ DEPORTES } \\
\hline \multirow[t]{2}{*}{ Universidad } & \multicolumn{2}{|c|}{ Básquetbol } & \multicolumn{2}{|c|}{ Vóleibol } & \multicolumn{2}{|c|}{$\begin{array}{c}\text { Tenis de } \\
\text { Mesa }\end{array}$} & \multicolumn{2}{|c|}{ Combate } & \multicolumn{3}{|c|}{ Totales } \\
\hline & $\mathrm{D}$ & $\mathrm{V}$ & $\mathrm{D}$ & $\mathrm{V}$ & $\mathrm{D}$ & $\mathrm{V}$ & $\mathrm{D}$ & $\mathrm{V}$ & $\mathrm{D}$ & $\mathrm{V}$ & $\mathrm{DV}$ \\
\hline PUCV & 6 & 8 & 5 & 8 & - & 2 & 6 & 8 & 17 & 26 & 43 \\
\hline UPLA & 7 & 7 & - & 3 & - & 2 & - & - & 7 & 12 & 19 \\
\hline USTM & 3 & 12 & 7 & 6 & - & 4 & - & 8 & 10 & 30 & 40 \\
\hline UV & - & - & 10 & 8 & - & - & 4 & 6 & 14 & 14 & 28 \\
\hline TOTAL & 16 & 27 & 22 & 25 & - & 8 & 10 & 22 & 48 & 82 & 130 \\
\hline$\%$ & \multicolumn{2}{|c|}{32,8} & \multicolumn{2}{|c|}{35,9} & \multicolumn{2}{|c|}{6,9} & \multicolumn{2}{|c|}{24,4} & \multicolumn{3}{|c|}{100} \\
\hline
\end{tabular}

$\mathrm{D}=$ Damas

TABLA I. Distribución de la población de acuerdo a las universidades y seleccionados de cada deporte.

$\mathrm{V}=\mathrm{V}$ arones

$\mathrm{DV}=$ Damas y Varones

Para este estudio se elaboro y validó un test para medir la capacidad de Percepción Subjetiva de Espacio (PSE), teniendo como base los fundamentos de los tests y ejercicios para el control de las capacidades coordinativas (1). La elaboración de las pruebas y posterior validez, se realizó en base a la literatura y consulta a profesionales y expertos en el área del aprendizaje motor, siguiendo principalmente una validez de contenido, en relación a todos los elementos de la variable que determina la PSE (2,3). Para determinar tanto los criterios primarios de la validez instrumental (Test), la objetividad y la confiabilidad, así como el de la situación experimental, en primer lugar se aplicó el test a deportistas pertenecientes a diferentes selecciones de un colegio particular de la $\mathrm{V}$ región, con un total de 30 alumnos. Los resultados obtenidos permitieron determinar que la Objetividad inter-observadores $(n=3)$, muestran un coeficiente de determinación de $100 \%$. Los datos del test y retest indican una la reproducibilidad de 0,92, lo cual muestra una alta consistencia.

\section{Test Percepción Subjetiva de Espacio (TPSE):}

Objetivo: Determinar a través de una distancia preestablecida la capacidad de PSE.

Descripción de la Situación Experimental:

Se coloca un cono en un lugar predeterminado de la cancha.

a. El evaluado se encuentra de pie al costado del cono.

b. El evaluado deberá recorrer trotando una distancia que se indicará en el momento.

c. El Test presenta dos situaciones de aplicación.

\section{Situación Experimental 1:}

1. a. Se le da a conocer la distancia en metros en que deberá realizar una trayectoria. Esta trayectoria se realizará trotando en línea recta desde un cono hacia la dirección que él estime conveniente. 
PAEZ H.J. Percepción subjetiva del espacio en deportistas seleccionados de las universidades tradicionales de la región de Valparaiso, Chile. Mot. Hum., 10(2): 103-111, 2010.

1. b. El evaluador pondrá el extremo (0) de una huincha metálica en la espalda del evaluado y tendrá en su poder el otro extremo. Esta distancia será medida desde el momento en que comienza a moverse desde el cono hasta que se encuentre detenido en el punto que él estime conveniente.

\section{Situación Experimental 2:}

2. a. El evaluado deberá indicar en metros la distancia en la cual él recorrerá la distancia .

2. b. El evaluador pondrá el extremo (0) de una huincha metálica en la espalda del estudiante sometido al test y tendrá en su poder el otro extremo para verificar la distancia que efectuará; esta distancia será medida desde el momento en que comienza a moverse desde el cono hasta que se encuentra detenido en el punto que él estime conveniente.

Para el análisis de los resultados, fue utilizada la estadística descriptiva, a través de la medida de tendencia central (promedio) y la medida de variabilidad (desviación estándar), además de representaciones graficas (gráficos de frecuencias) y porcentajes.

Para responder las hipótesis de esta investigación, se utilizó la estadística inferencial, específicamente la prueba t de student

\section{RESULTADOS}

Los datos brutos del test de Percepción Subjetiva de Espacio (TPSE) con tiempo determinado por el evaluador y tiempo determinado por el evaluado de esta investigación son presentados en la TABLA II.

\begin{tabular}{|c|c|c|c|c|c|c|c|}
\hline \multirow{4}{*}{ DEPORTES } & \multicolumn{6}{|c|}{ PERCEPCIÓN SUBJETIVA DE ESPACIO } & \\
\hline & \multirow{3}{*}{$\begin{array}{c}\text { ESTD. } \\
\text { X }\end{array}$} & \multicolumn{2}{|c|}{ FEMENINO } & \multicolumn{2}{|c|}{ MASCULINO } & \multicolumn{2}{|c|}{ TOTAL } \\
\hline & & PSE-1 & PSE-2 & PSE-1 & PSE-2 & PSE 1 & PSE 2 \\
\hline & & $-1,59$ & 1,03 & $-1,12$ & 0,95 & 1,3 & 0,98 \\
\hline \multirow[t]{4}{*}{ BASQUETBOL } & $\mathrm{S}$ & 1,36 & 1,274 & 1,29 & 0,95 & 1,337 & 1,08 \\
\hline & $\Sigma$ & $-25,55$ & $-16,58$ & $-30,39$ & $-25,73$ & 55,94 & 42,31 \\
\hline & $\mathrm{N}$ & 16 & 16 & 27 & 27 & 43 & 43 \\
\hline & $\mathrm{X}$ & $-1,49$ & $-3,54$ & $-0,73$ & $-0,52$ & 1,09 & 0,91 \\
\hline \multirow[t]{4}{*}{ VOLEIBOL } & $\mathrm{S}$ & 1,21 & 1,53 & 0,87 & 0,631 & 1,251 & 1,218 \\
\hline & $\Sigma$ & $-32,98$ & $-29,8$ & $-18,33$ & $-13,17$ & 51,31 & 42,97 \\
\hline & $\mathrm{N}$ & 22 & 22 & 25 & 25 & 47 & 47 \\
\hline & $X$ & & & $-1,71$ & $-1,28$ & 1,71 & 1,28 \\
\hline TENIS & $\mathrm{S}$ & & & 0,897 & 0,712 & 0,9 & 0,75 \\
\hline \multirow[t]{3}{*}{ DE MESA } & $\Sigma$ & & & $-13,75$ & $-10,26$ & 13,75 & 10,26 \\
\hline & $\mathrm{N}$ & & & 8 & 8 & 8 & 8 \\
\hline & $\mathrm{X}$ & 0,82 & $-0,77$ & $-0,61$ & $-0,5$ & 0,68 & 0,58 \\
\hline DEPORT. & $\mathrm{S}$ & 0,77 & 0,714 & 0,94 & 0,75 & 0,897 & 0,71 \\
\hline \multirow[t]{3}{*}{ COMBATE } & $\Sigma$ & $-8,21$ & $-7,76$ & $-13,2$ & -11 & 21,81 & 18,76 \\
\hline & $\mathrm{N}$ & 10 & 10 & 22 & 22 & 32 & 32 \\
\hline & $\mathrm{X}$ & 1,39 & $-0,73$ & 0,92 & $-1,35$ & $-1,09$ & $-4,33$ \\
\hline \multirow[t]{3}{*}{ TOTAL } & $\mathrm{S}$ & 1,22 & 0,83 & 1,1 & 1,24 & 1,17 & 1,46 \\
\hline & $\Sigma$ & 66,74 & $-54,14$ & 76,07 & $-60,16$ & $-142,8$ & $-536,4$ \\
\hline & $\mathrm{N}$ & 48 & 48 & 82 & 82 & 130 & 130 \\
\hline
\end{tabular}

TABLA II. Distribución de los resultados del test Percepción Subjetiva de Espacio (PSE-1 y PSE 2) por Deportes, según sexo.PSE-1= Percepción Subjetiva Espacio, determinado por el Evaluador.

PSE-2= Percepción Subjetiva Espacio, determinado por el Evaluado.

$\mathrm{X}=$ Promedio

$\mathrm{S}=\quad$ Desviación estándar

$\Sigma=$ Suma 
PAEZ H.J. Percepción subjetiva del espacio en deportistas seleccionados de las universidades tradicionales de la región de Valparaiso, Chile. Mot. Hum., 10(2): 103-111, 2010.

Los datos del test de Percepción Subjetiva de Espacio son presentados a través de una estadística descriptiva, usando para ello, una representación iconográfica.

La Fig. 1 presenta las diferencias de los promedios totales en los tests PSE-1 y PSE-2 según sexo. Se puede observar que las damas obtienen mejores desempeños en el test PSE-2 con una diferencia de sus promedios de $0,65 \mathrm{~m}$., situación que no se repite en los varones donde éstos obtienen mejores resultados en el TPSE-1, siendo la diferencias de sus promedios de 0,43. Es decir, cuando los seleccionadas le indican su tiempo de ejecución (PST-2), poseen un mejor desempeño, situación que no se repite con los varones ya que éstos poseen mejor desempeño cuando el espacio a recorrer lo indican ellos.

Por otro lado, en el TPSE-1 los varones obtienen mejor desempeño que las damas con una diferencia entre éstos de 0,47 m., y en el TPSE-2 las damas obtienen un mejor desempeño con 0,61 m., en la diferencias de sus promedios.

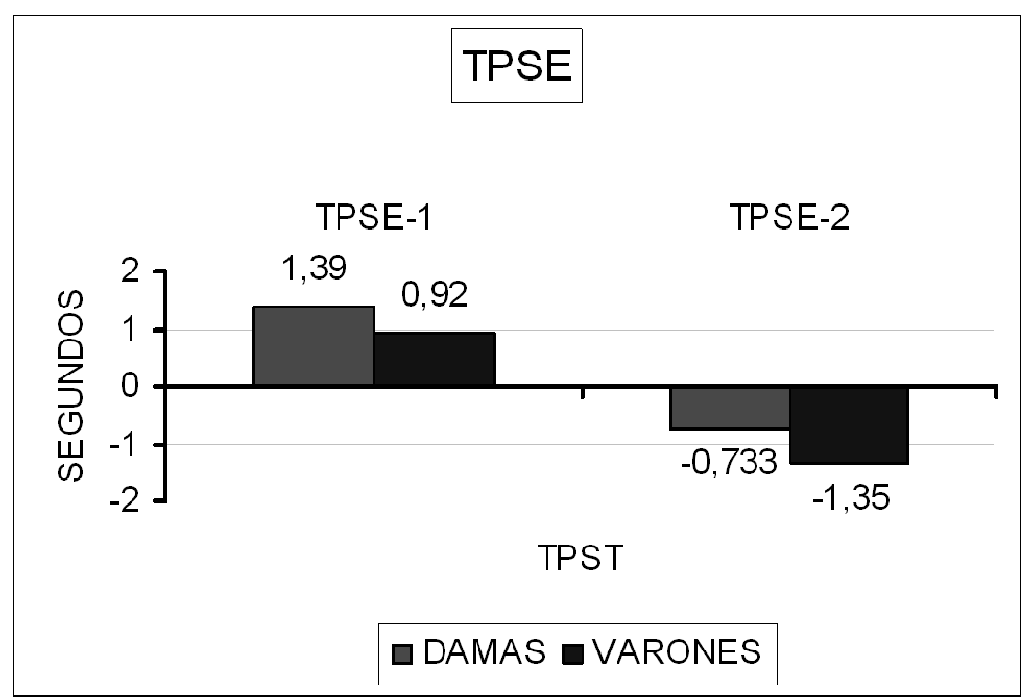

Figura 1. Representación gráfica de los desempeños de damas y varones en el test PSE-1 y PSE-2 (en metros).

La Fig. 2 exhibe los promedios de los deportistas de deportes Colectivos e Individuales en el TPSE-1 y TPSE-2, donde se puede estimar que éstos obtienen mejores resultados en el TPSE-2, lo que significa que presentan un desempeño superior cuando la distancia a ejecutar es determinada por ellos, presentando en los deportes colectivos una diferencia de sus promedios de $-0,24 \mathrm{~m}$., y en los deportes individuales de $-0,13$ m., en relación al TPSE-1.

En el TPSE-1 presentan mejores desempeños los deportes individuales con una diferencia de sus promedios de $-0,31 \mathrm{~m}$., situación que se repite en el TPSE-2 con una diferencia de sus promedios de$0,19 \mathrm{~m}$. 
PAEZ H.J. Percepción subjetiva del espacio en deportistas seleccionados de las universidades tradicionales de la región de Valparaiso, Chile. Mot. Hum., 10(2): 103-111, 2010.

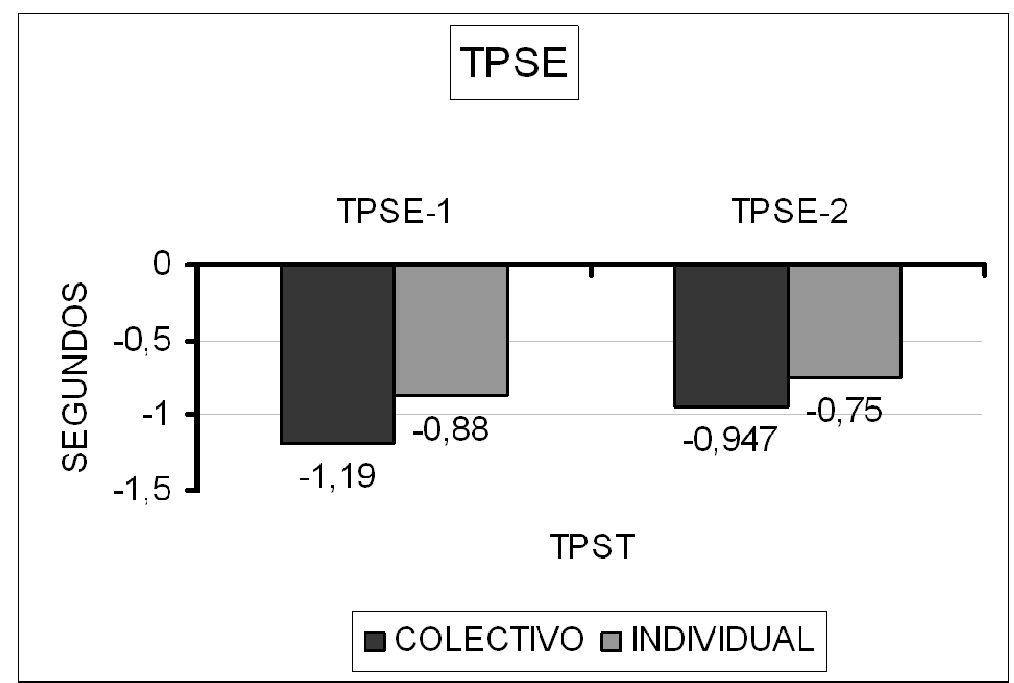

Figura 2. Representación gráfica de los desempeños de deportes colectivos e individuales en el test PSE-1 y PSE-2 (en metros)

Se presentan a continuación los desempeños de los seleccionados de los deportes colectivos de Básquetbol y Voleibol (Fig.3) en los tests de PSE-1 y PSE-2 en donde se puede apreciar que los seleccionados del basquetbol presentan mejores desempeños en el TPSE-2 lo que significa que tienen mejores resultados cuando la distancia a recorrer es determinada por ellos, presentando una diferencia de sus promedios de 0,32 m.; por otro lado, en el caso de los seleccionados del Voleibol esta situación no se repite ya que ellos presentan mejores desempeños cuando la distancia a recorrer les es determinada, presentando una diferencia de sus promedios de 2,2 $\mathrm{m}$.

En el TPSE-1, los seleccionados del Voleibol presentan mejores desempeños que los seleccionados del Básquetbol, presentando una diferencia de sus promedios de $0,21 \mathrm{~m}$., situación que no se repite en el TPSE-2 donde los seleccionados del básquetbol presentan mejor desempeño que los seleccionados de Vóleibol, presentando una diferencia de sus promedios de $2,31 \mathrm{~m}$.

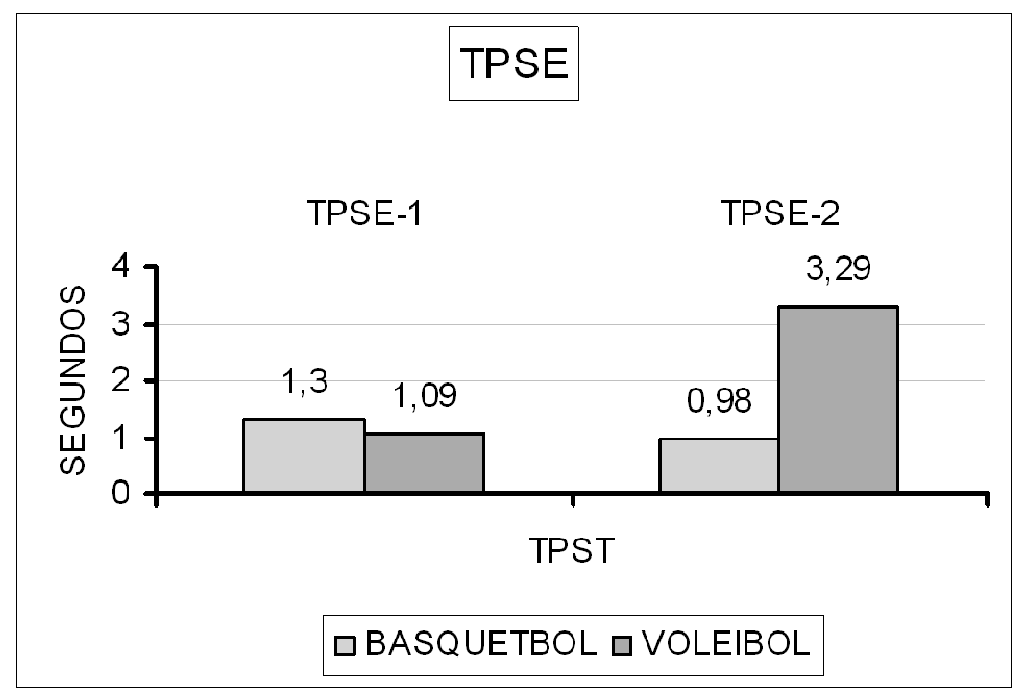

Figura 3. Representación gráfica de los desempeños de deportes Colectivos de Básquetbol y Voleibol en el test PSE 1 y 2 (en segundos). 
PAEZ H.J. Percepción subjetiva del espacio en deportistas seleccionados de las universidades tradicionales de la región de Valparaiso, Chile. Mot. Hum., 10(2): 103-111, 2010.

En la Fig. 4 se presentan los desempeños de los seleccionados (as) de los deportes individuales, tenis de mesa y deportes de combate en los test de PSE-1 y PSE-2 en donde se puede apreciar que los seleccionados de tenis de mesa presentan mejores desempeños en el TPSE-1, lo que significa que poseen mejores resultados cuando la distancia a ejecutar les es indicada, presentando una diferencia de sus promedios de 0,6 m., situación que no se repite en el caso de deportes de combate ya que éstos presentan mejores desempeños cuando la distancia a recorrer es indicada por ellos, siendo la diferencia de sus promedios de $1,13 \mathrm{~m}$.

En el TPSE-1, los seleccionados de tenis de mesa presentan mejores desempeños que los de deportes de combate, presentando una diferencia de sus promedios de 0,02 m., no así en el TPSE-2 donde los mejores resultados los presentan los seleccionados de deportes de combate con una diferencia de sus promedios de $0,7 \mathrm{~m}$.

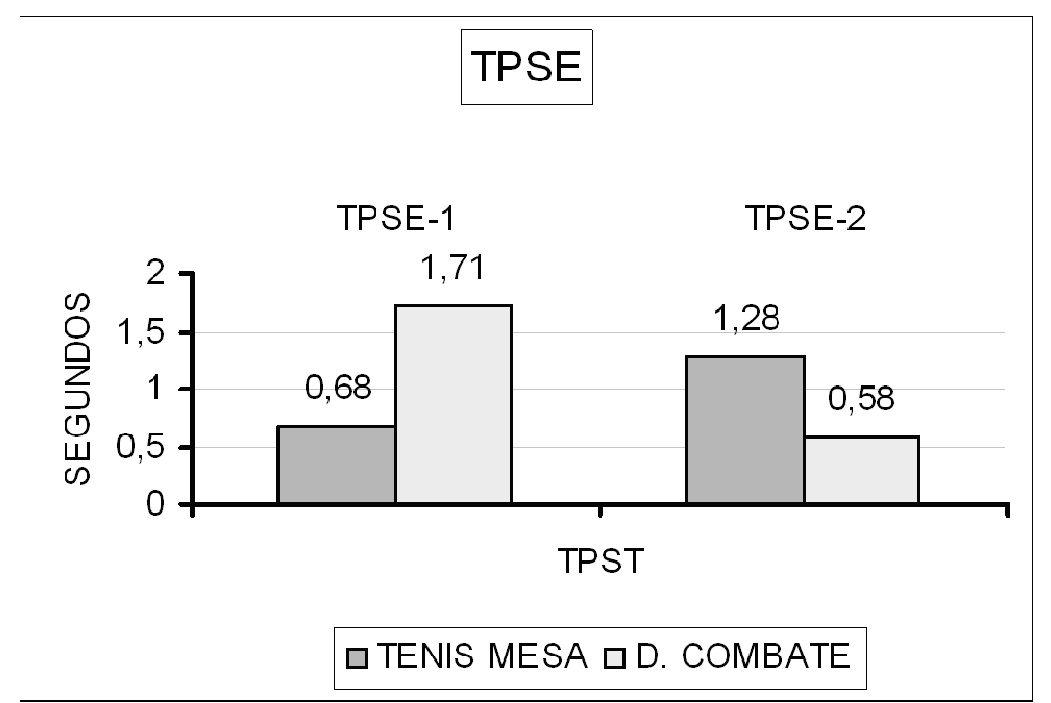

Figura 4. Representación gráfica de los desempeños de deportes Individuales de Tenis de Mesa y deportes de Combate en el test PSE 1 y 2 (en metros).

\section{DISCUSIÓN}

A partir de los datos expuestos y con el fin de poder responder a los objetivos planteados en este estudio, se puede argumentar que la capacidad de percepción subjetiva de espacio está presente en cada acción motriz que se realice. Cabe señalar que esta capacidad es esencial para poder realizar cualquier toma de decisión durante la acción deportiva y realizar una efectiva anticipación, es por esto, que se les atribuye gran importancia en el rendimiento deportivo.

En relación a los desempeños entre los varones y las damas, sobre la capacidad de percepción subjetiva de espacio. Los seleccionados universitarios presentan un mejor desempeño en la ejecución en relación a las seleccionadas universitarias; por lo tanto, se podría manifestar que una respuesta motora de estos en cualquier situación deportiva, en que se requiera esta capacidad, tendrían mayor acierto en sus respuestas, y por lo tanto, un mayor rendimiento deportivo que las damas.

Afirmando los resultados de este estudio, Antúnez (4) se refiere a las diferencias de los varones sobre las damas, y la superioridad de éstos en las prácticas deportivas, en cuanto a que el rendimiento deportivo de los varones está marcado no sólo por las diferencias biológicas, sino que se incrementan con las diferencias psicológicas, sociológicas y culturales,

Sillero (5), evaluó la percepción de las trayectorias como tarea visual, en 189 sujetos y demostró que los varones obtienen mejor eficacia, en la calidad de 
PAEZ H.J. Percepción subjetiva del espacio en deportistas seleccionados de las universidades tradicionales de la región de Valparaiso, Chile. Mot. Hum., 10(2): 103-111, 2010.

toma de decisiones, anticipación, aciertos y coeficiente de percepción de trayectoria en relación al desempeño de las damas.

En relación a que los deportes individuales presentan mejor desempeño en la percepción subjetiva de espacio en relación a los deportes colectivos.

Se han obtenido resultados no del todo coincidente como los estudios de Sillero \& Rojo (6), evaluaron la percepción de trayectoria, combinación de la precisión y la anticipación de la toma de decisiones en 98 sujetos, determinando que estas capacidades son mejores en los deportistas que participan en deportes con balón.

En el deporte Básquetbol los varones presentan un mejor desempeño en el test perceptivo de de espacio en relación a las damas. Al respecto, Sillero (5) señala que los varones que actuaban deportivamente con balón presentaban un mejor desempeño que las damas; además Montaner \& Montaner (7), realizaron un estudio comparativo del tiempo de posesión y sus efectos en un equipo de baloncesto, señalando que los varones juegan posesiones más largas que las damas y presentan mayor efectividad en todos los intervalos de tiempo.

Entre estos seleccionados encontramos que los varones tienen mayor tiempo de práctica en este deporte que las damas, factor que puede ser incidente en este resultado. Al respecto, un estudio realizado por Sillero (5), sobre la percepción de trayectorias como tarea visual, en 189 sujetos, señaló la importancia de la experiencia deportiva acumulada, logrando una mejor anticipación y coeficiente de aciertos los de mayor experiencia deportiva, señalando que la percepción de trayectoria es una habilidad que se mejora con la práctica. Por otro lado, Gómez (8) realizó un análisis de los procesos perceptivos y de toma de decisiones en jugadores cadetes de baloncesto, señalando que los jugadores experimentados presentan menores posiciones de fallo perceptivo, cometen menos errores, tienen mayor variedad en la toma de decisiones y variabilidad en sus acciones de juego.

\section{CONCLUSIONES}

Serán presentadas siguiendo una estrategia de respuesta tanto para los objetivos como para las hipótesis, y que sintetizan de la siguiente manera:

\section{Según los objetivos planteados:}

Se puede Diseñar y validar una prueba de Percepción Subjetiva de Espacio que permita medir en una situación real a los deportistas. En este sentido se diseño y valido el test de Percepción Subjetiva de Espacio (PSE), a través de una validez de contenido, con un coeficiente de determinación de un cien por ciento de objetividad, de interobservadores y una alta consistencia del índice de reproductibilidad.

Se puede Identificar qué género presenta mejores desempeños en la capacidad de percepción subjetiva de espacio en cada una de la pruebas con distancia fijo y por determinar. Los varones presentan mejores desempeños que las damas en la PSE-1 (cuando se le da la distancia), situación que no se repite en el test PSE-2 (cuando él o ella decide la distancia) donde las damas obtienen un mejor desempeño.

Se puede Determinar si los deportes colectivos presentan mejores desempeños en la capacidad de percepción subjetiva de espacio en cada una de la pruebas con distancia fija y distancia por determinar con respecto a los deportes individuales. Los resultados indican que los deportes individuales obtienen un mejor desempeño que los deportes colectivos en el test de percepción subjetiva de espacio.

Se puede Identificar qué deporte colectivo presenta mejores desempeños en la capacidad de percepción subjetiva tiempo y espacio en cada una de la pruebas con tiempo fijo y sin tiempo fijo. En general, los deportistas seleccionados de Vóleibol presentan los mejores desempeños en el test PSE-1, que los seleccionados de Básquetbol, resultado que no se repite en el test PSE-2.

Se puede Identificar qué deporte individual presenta mejores desempeños en la capacidad de percepción subjetiva de tiempo y de espacio en cada una de la pruebas con tiempo fijo y sin tiempo fijo. Los deportistas seleccionados de tenis de mesa presentan 
PAEZ H.J. Percepción subjetiva del espacio en deportistas seleccionados de las universidades tradicionales de la región de Valparaiso, Chile. Mot. Hum., 10(2): 103-111, 2010.

un mejor desempeño en el test de PSE-1 que los seleccionados de deportes de combate, y éstos últimos en el test PSE-2.

\section{Según las hipótesis planteadas:}

Los deportistas universitarios seleccionados presentan diferencias significativas entre los desempeños de los test PSE-1 y PSE-2, $(\alpha 0,05)$. Aplicada la prueba $t$ student a los resultados, esta hipótesis es rechazada, es decir, no existen diferencias significativas en los desempeños de los deportistas universitarios seleccionados entre los desempeños de ambos tests.

Los deportistas universitarios seleccionados damas presentan diferencias significativas entre los desempeños de los test PSE-1 y el test PSE-2 en relación a los deportistas varones, $(\alpha 0,05)$. Aplicada la prueba $t$ student a los resultados del test PSE-1, esta hipótesis es rechazada, es decir no existen diferencias significativas en las deportistas seleccionadas damas en los desempeños de los test PSE-1 en relación a los deportistas varones. Situación que no se repite en el test PSE-2 donde aplicada la prueba $t$ student a los resultados, esta hipótesis es aceptada, es decir, existen diferencias significativas en los desempeños de las deportistas seleccionadas damas en relación a los varones.

Los deportistas universitarios seleccionados de deportes colectivos presentan diferencias significativas entre los desempeños de los test PSE1 y el test PSE-2 en relación a los deportistas de deportes individuales, $(\alpha 0,05)$. Aplicada la prueba t student a los resultados, esta hipótesis es rechazada, es decir no existen diferencias significativas en los desempeños de los deportistas universitarios seleccionados de deportes colectivos en ambos tests, en relación a los deportistas de deportes individuales.

Existen diferencias significativas entre los deportistas seleccionados de deportes colectivos de básquetbol y vóleibol en los test PSE-1 y PSE-2 ( $\alpha$ $0,05)$. Aplicada la prueba $t$ student a los resultados del los test PSE-1 y 2, ésta es rechazada, es decir, no existen diferencias significativas en los desempeños de los deportistas universitarios de ambos deportes colectivos.

Existen diferencias significativas entre los deportistas seleccionados de deportes individuales de tenis de mesa y deportes de combate en los test PSE-1 y PSE-2, $(\alpha 0,05)$. Aplicada la prueba $\mathrm{t}$ student a los resultados del test PSE-1, es decir, existen diferencias significativas en los desempeños de los deportistas universitarios de deportes individuales de tenis de mesa y deportes de combates.

\section{REFERENCIAS BIBLIOGRAFICAS}

1. Jung $\mathrm{R}$, Wilkner $\mathrm{H}$. Testes e exercícios para controle das capacidades coodenativas. Revista de Educação Física e Desporto. Horizonte, 1987, $\mathrm{IV}(20)$, 53-57.

2. Barbanti V. (2003). Dicionário de Educação Física e do Esporte. (2.ed.). São Paulo: Edit. Manole.

3. León O, Montero I. (2002). Métodos de Investigación en psicología y Educación. (3.ed.). Madrid: Edit. McGraw-Hill / Interamericana, España.

4. Antúnez M. (2001). Reflexiones acerca de lo que la mejora representa para el deporte y el verdadero significado del deporte para la mujer. [on line]. Disponible: http://www.efdeportes.com/efd42/mujer.htm [11 Junio 2002].

5. Sillero M. (2002). Percepción de trayectorias como tarea visual. Propuesta de evaluación en el futbol. Tesis (Doctoral). Universidad Politécnica de Madrid. Madrid.

6. Sillero M, Rojo J. Percepción de trayectorias de balones entre los 9 y 8 años. APUNTS Educación Física y Deportes, 2001 (66), 40-43.

7. Montaner C, Montaner A. (2004). Estudio comparativo del tiempo de posesión y sus efectos en el juego entre un equipo masculino y uno femenino de baloncesto de elite. [on line] Disponible:

http://www.RendimientoDeportivo,com/N009/art ic045.htm.

8. Gómez M. (2006). Análisis de los procesos perceptivos y de toma de decisiones en jugadores cadetes de balonmano. [on line]. Disponible: http://www.efdeportes.com/efd95/balonc.htm. 
PAEZ H.J. Percepción subjetiva del espacio en deportistas seleccionados de las universidades tradicionales de la región de Valparaiso, Chile. Mot. Hum., 10(2): 103-111, 2010.

\begin{abstract}
Subjective Perception of Space (PSE) has been considered one of the determinants of the performance capabilities of sports, which is reflected on a game development faster and more accurate in an environment of high levels of temporal and spatial uncertainty. That is why this study aims to determine the level of PSE in two different situations, college athletes selected in the $V$ Region. The research is descriptive, with an empirical-analytic paradigm. The population consisted of $N=130$ athletes $(n=48$ women $n=82$ males). To determine the dependent variable PSE test was used with a fixed distance mode and distance to be determined. The main results after application of the student t test indicate that: a) no significant differences in college athletes test the performances of PSE-1 and PSE-2. b) in the TPSE-2 in relation to the comparison $\begin{array}{llll}\text { of sexes } & \text { significant } & <0.05)\end{array}$
\end{abstract}

Keywords: Subjective Perception, Time, Space, Sports, University.

Dirigir correspondencia a:

Jacqueline del Carmen Paez Herrera

Pontíficia Universidad Católica de Valparaíso

Avda. El Bosque 1290, santa Inés, Viña del Mar

CHILE,

jp.atletismopucv@hotmail.com

RECIBIDO 20-10-2010

ACEPTADO 17-12-2010 